\title{
Evaluación de la Competencia Transversal 2: Aplicación y pensamiento práctico
}

\section{Vallada, Eva a ; Martín, Jorge ${ }^{a}$; Úbeda, Joan Enric ${ }^{\mathrm{b}}$ y Crespo Fortunato ${ }^{\mathrm{a}}$}

aDepartamento de Estadística e Investigación Operativa Aplicdas y Calidad; Universitat Politècnica de València; \{evallada,jmartinm,fcrespo\}@eio.upv.es; ${ }^{b}$ Departamento de Economía y Ciencias Sociales. Universitat Politècnica de València, juaubgar@esp.upv.es

\begin{abstract}
En este trabajo se presenta la competencia transversal 2 evaluada en la Universitat Politècnica de València, "Aplicación y Pensamiento Práctico". En concreto se centra en la evaluación de dicha competencia en 3 asignaturas del Grado en Administración y Dirección de Empresas que se imparte en dicha Universidad. Las asignaturas corresponden tanto al nivel uno (primer y segundo curso) como al dos (tercer y cuarto curso) de la titulación.
\end{abstract}

Keywords: , aplicación y pensamiento práctico, evaluación.

\section{Resumen}

En este trabajo se presenta la competencia transversal 2 evaluada en la Universitat Politècnica de València, "Aplicación y Pensamiento Práctico". En concreto se centra en la evaluación de dicha competencia en 3 asignaturas del Grado en Administración y Dirección de Empresas que se imparte en dicha Universidad. Las asignaturas corresponden tanto al nivel uno (primer y segundo curso) como al dos (tercer y cuarto curso) de la titulación.

Palabras clave: competencias, aplicación y pensamiento práctico, evaluación. 


\section{Introducción y Objetivos}

Tanto graduados como empleadores consideran de gran importancia en el desempeño profesional de los titulados la formación en competencias no solo específicas, sino generales o transversales.

La UNESCO, en la Conferencia Mundial sobre Educación Superior de París de 1998, señaló como uno de los ejes prioritarios la formación en competencias (Arroyo-Cañada, 2019), por lo que cada vez son más las Universidades que consideran prioritaria la evaluación por competencias (Delors, 1996). El objetivo es que los/las egresados/as adquieran un perfil competencial y común a todas las titulaciones.

Así, las denominadas Competencias Transversales tienen un indudable valor en el desempeño profesional de los titulados universitarios. En este sentido la Universitat Politècnica de València (UPV) ha puesto en marcha un Proyecto institucional para incluir de forma explícita la exigencia de que nuestros estudiantes se formen en estas competencias y sean evaluados para poder ser acreditado su nivel de dominio (UPV, 2019). El objetivo es que los/las egresados/as adquieran un perfil competencial y común a todas las titulaciones.

Considerando normativas y directrices tanto nacionales como internacionales (ANECA, 2019), en la Universitat Politècnica de València (UPV) se evalúan un total de 13 Competencias Transversales, fundamentales para que nuestros/as estudiantes finalicen sus grados preparados para afrontar y resolver los problemas de nuestro entorno real. En concreto, las competencias a evaluar son:

CT1: Compresión e integración

CT2: Aplicación y Pensamiento Práctico

CT3: Análisis y Resolución de Problemas

CT4: Innovación, Creatividad y Emprendimiento

CT5: Diseño y Proyecto

CT6: Trabajo en Equipo y Liderazgo

CT7: Responsabilidad Ética, Medioambiental y Profesional

CT8: Comunicación Efectiva

CT9: Pensamiento Crítico

CT10: Conocimiento de Problemas Contemporáneos

CT11: Aprendizaje Permanente

CT12: Planificación y Gestión del Tiempo

CT13: Instrumental Específica 
Las calificaciones que se pueden obtener en cada una de las competencias son las siguientes:

- A: excelente

- B: adecuada

- C: en desarrollo

- D: no alcanzada

Cabe destacar que cada una de las 13 competencias se debe evaluar como mínimo dos veces en cada nivel, es decir, dos veces en distintas asignaturas de primer y segundo curso y otras dos veces en distintas asignaturas de tercer y cuarto curso.

El presente trabajo se centra en la CT2: Aplicación y Pensamiento Práctico, que según se indica en http://www.upv.es/contenidos/COMPTRAN/info/954715normalc.html, tiene como objetivo "Aplicar los conocimientos teóricos y establecer el proceso a seguir para alcanzar determinados objetivos, llevar a cabo experimentos y analizar e interpretar datos para extraer conclusions."

Esta competencia está muy relacionada con el hecho de que los/las estudiantes deben saber aplicar todos los conocimientos teóricos adquiridos durante 4 años a un entorno real. Esta adaptación a los problemas reales es fundamental, ya que se deben tomar decisiones y proponer soluciones en entornos donde los recursos serán escasos. Debe centrarse en un modo de pensar dirigido a la acción, que permita adaptarse a nuevas situaciones y tomar decisiones, es decir, Actuar.

El objetivo principal de este trabajo es mostrar como se evalúa la CT2 en varias asignaturs del Grado en Administración y Direccón de Empresas. Con esto se pretende que profesores de distintas asignaturas comparen métodos de evaluación y puedan surgir sinergias entre ellos. En concreto, se presentan las formas de evaluación de la CT2 en tres asignaturas:

- Métodos Estadísticos en Economía (nivel 1)

- Gestión de Calidad (optativa de nivel 2)

- Banca y Bolsa (optative de nivel 2)

\section{Asignaturas seleccionadas}

Como ya se ha comentado, en este trabajo se presentan las actividades formativas y las formas de evaluación de tres asignaturas. En la Tabla 1 podemos ver los detalles de las tres asignaturas.

Tabla 1. Detalle de asignaturas

\begin{tabular}{|ccccc|}
\hline Asignatura & Tipo & Curso & Cuatrimestre & Créditos \\
\hline Métodos Estadísticos en Economía & Formación Básica & 2 & A & 6 \\
Gestión de Calidad & Optativa & 4 & A & 4,5 \\
Banca y Bolsa & Optativa & 4 & A & 4,5 \\
\hline
\end{tabular}


Fuente: Elaboración propia a partir de la información en http://www.upv.es/titulaciones/GADE/

Los procedimientos de evaluación para la CT2 que sugiere la UPV en http://www.upv.es/contenidos/COMPTRAN/info/954721normalc.html son los siguientes:

- Resolución de problemas, desde sencillos a más complejos donde no solo se considere el resultado, sino el procedimiento para resolverlo.

- Observación de ejecuciones prácticas.

- Redacción de informes, donde se pueda evaluar el proceso de pensamiento aplicado por el/la estudiante.

- Resolución de casos.

- Elaboración de proyectos.

Respecto a las actividades formativas para adquirir esta competencia, la UPV propone las siguientes:

- Actividades grupales.

- Estudio de casos.

- Exposiciones orales.

- Foros y debates.

- Juego y simulación.

- Prácticas de laboratorio.

- Preguntas.

- Problemas.

- Proyectos.

- Redacción de informes.

- Visitas externas

Teniendo en cuenta las sugerencias de la UPV, en los siguientes puntos se pasa a explicar las actividades formativas y la forma de evaluar la CT2 por parte de las asignaturas consideradas en este trabjo.

\section{Evaluación de la Competencia Transversal 2}

Tal y como se ha comentado, esta competencia hace referencia a cubrir el "salto" o "gap" que existe entre la teoría y la práctica. El objetivo es que los/las estudiantes estén preparados para ello al finalizar sus estudios de Grado. En los siguientes puntos se procede a detallar las actividades formativas y la evaluación propuestas por cada una de las asignaturas. 


\section{Métodos Estadísticos en Economía}

La asignatura de Métodos Estadísticos en Economía está ubicada en el Nivel 1 de la titulación, en concreto en el primer cuatrimestre de segundo curso. Es una asignatura de 6 créditos donde se forma a los estudiantes en la realización de análisis estadísticos utilizando diferentes herramientas. Cabe destacar que esta asignatura es continuación de Introducción a la Estadística, asignatura que se imparte en primer curso.

Las actividades formativas de la CT2 en esta asignatura están centradas principalmente en la resolución de problemas, prácticas de laboratorio y redacción de informes.

Respecto a la evaluación de la CT2 se realiza a través de la resolución de problemas, en concreto los problemas que aparecen en la prueba de evaluación individual de la asignatura. En dicha prueba, se informa a los estudiantes sobre los problemas que corresponden a la evaluación de la competencia y se considera no solo el resultado final, también el procedimiento para llegar al mismo.

\subsection{Gestión de Calidad}

La asignatura de Gestión de Calidad está ubicada en el Nivel 2 de la titulación, en concreto en el primer cuatrimestre de cuarto curso. Es una asignatura de 4,5 créditos donde se forma a los estudiantes en los diferentes modelos de gestión de calidad con el objetivo de que sean capaces de desarrollar, gestionar y evaluar sistemas de calidad en las organizaciones empresariales.

Las actividades formativas utilizadas en esta asignatura para la CT2 se centran principalmente en la elaboración de informes, estudio de casos y actividades en grupo.

En el caso de la evaluación de la CT2 se realiza a través de un trabajo grupal. Este trabajo supone un $70 \%$ de la nota final y los/las estudiantes deben aplicar los conceptos de Gestión de Calidad a una empresa seleccionada por ellos/as y cercana a su entorno. El trabajo se realiza en grupos de 4 personas y se realiza una exposición oral, donde el profesor puede comprobar el grado de implicación de cada miembro del grupo.

Dependiendo del trabajo presentado, se le evaluará la CT2, como es difícil diferenciar la calificación para cada miembro del grupo (ya que la calificación del trabajo es conjunta) se matiza la evaluación de la CT2 a partir de la exposición oral y de las consultas sobre aspectos del trabajo realizadas por los miembros del grupo a lo largo del curso. Generalmente, con una evaluación del trabajo por encima del 7.5 se califica la CT2 con la A, cuando la nota del trabajo está entre el 5 y el 7,5 con una B y si la nota del trabajo es inferior a 3 puntos, la CT2 se califica con una D.

\subsection{Banca y Bolsa}

La asignatura de Banca y Bolsa está ubicada en el Nivel 2 de la titulación, en concreto en el primer cuatrimestre de cuarto curso. Es una asignatura de 4,5 créditos donde se forma a los estudiantes en los dos bloques que conforman la asignatura. En el de Banca los/las estudiantes analizan la actual estructura del sistema bancario español y los principales 
retos durante los próximos años, teniendo en cuenta su evolución histórica. Respecto al bloque de Bolsa, se introduce al estudiante en la formación de carteras y el binomio rentabilidad/riesgo, así como en el análisis fundamental y técnico del mercado de valores.

Respecto a las actividades formativas utilizadas en esta asignatura para la CT2, se centran principalmente en las actividades grupales, foros y debates y prácticas de laboratorio.

En el caso de la evaluación, la CT2 es evaluada a través de dos actividades:

- Sesión de prácticas. En una de las sesiones de prácticas se abre un debate/foro donde los/las estudiantes indican cómo han utilizado los distintos productos bancarios durante la última semana. De qué manera han consumido y pagado y las razones por las que se utilizan unos sistemas de pago más que otros. El objetivo es que analicen su comportamiento como consumidores y lo relacionen con los contenidos vistos en la asignatura. En otra sesión, se aborda la problemática de la crisis financiera contemporánea, su origen e implicaciones a partir del análisis de materiales como informes técnicos publicados por organismos oficiales $y$ gabinetes técnicos.

- Casos prácticos. Se realizan dos trabajos que consisten por una parte en estudiar alguna entidad de su entorno cercano, como pueden ser las oficinas bancarias de su barrio o pueblo. El objetivo es que analicen la evolución histórica de las entidades seleccionadas. En este caso no se realiza exposición oral por falta de tiempo para ello. En el otro caso práctico se aborda la composición de una cartera bursátil durante un período de tiempo determinado, solicitando a las y los estudiantes una valoración de los resultados alcanzados.

En ambos casos se solicita a las y los estudiantes que apliquen los conocimientos adquiridos en otras asignaturas a lo largo de su formación, así como la aplicación de los conocimientos teóricos formulados en clase para poder alcanzar los objetivos planteados para las actividades señaladas.

Los resultados de la evaluación en esta asignatura evidencian que el nivel de competencia transversal evaluada alcanzado en cada una de las actividades se correlacionan positivamente con la calificación académica obtenida en la misma.

\section{Conclusiones}

La formación en competencias transversales es fundamental para que los/las estudiantes en general y en particular los de la Universitat Politècnica de València finalicen sus estudios bien preparados para ejercer como profesionales en el mundo real. De las 13 competencias transversales que se evalúan en la UPV, este trabajo se centra en la segunda, «Aplicación y Pensamiento Práctico». El objetivo de esta competencia es que los alumnos sepan trasladar los conceptos vistos en los 4 años que dura el Grado a un entorno real, es decir, estar formados para desempeñar su vida profesional de la mejor manera posible. En concreto, se han analizado las actividades formativas y la manera de evaluar la 
competencia por parte de 3 asignaturas, una de ellas de formación básica y las otras dos optativas. Se ha podido ver que en todos los casos se utilizan actividades de formación y métodos de evaluación recomendados para esta competencia por la UPV.

\section{Referencias}

ANECA (2019). Guía de apoyo para la redacción, puesta en práctica y evaluación de los resultados del aprendizaje. Versión 1.0. ANECA. http://www.aneca.es/Documentos-ypublicaciones [14 mayo 2019].

ARROYO-CAÑADA, F-J. (2019). "La evaluación por competencias en estudios empresariales" en Journal of Management and Business Education, vol 2(1), p. 1-7

DELORS, J. (Presidente de la Comisión) (1996). La educación encierra un tesoro. Informe a la Unesco de la Comisión Internacional sobre la Educación para el siglo XXI. http://unesdoc.unesco.org/images/0010/001095/109590so.pdf [mayo 2019].

UPV. (2019). Proyecto institucional de las competencias transversales UPV. http://www.upv.es/competenciastransversales [ junio 2019] 